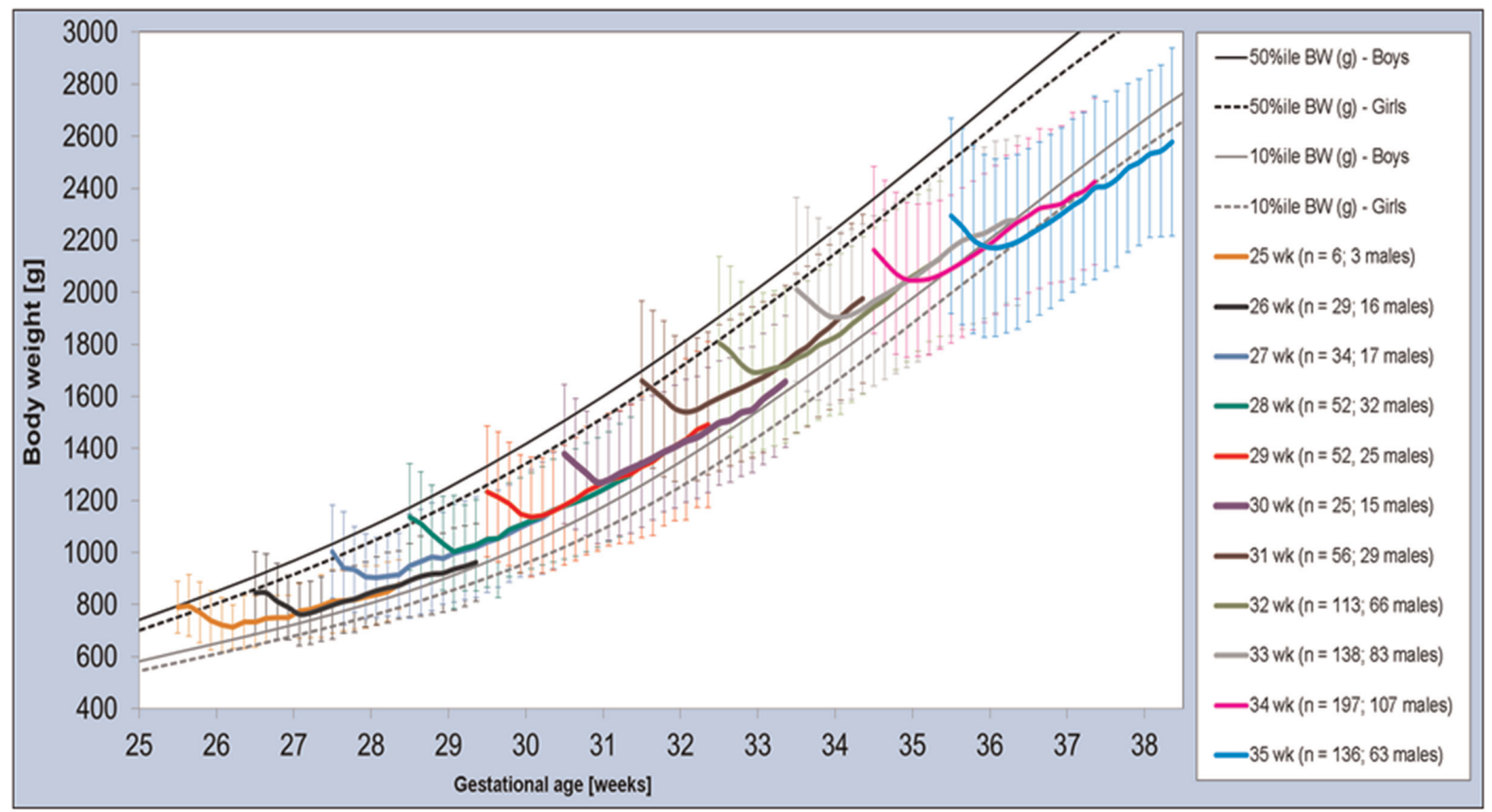

Abstract P0-0460 Figure 1 Preterm FFMI increases progressively until 40 weeks, then remains constant over time like terms

FFM and FM can define nutritional depletion or obesity. We present longitudinal data from a large dataset of stable growing infants.

Methods BC data $(\mathrm{n}=857)$ from 574 infants $(22-42$ wks $)$ enrolled in 4 longitudinal studies, $2 / 3 \mathrm{rd}$ were preterm, was considered as independent data points. Preterms on fortified breast milk or formula $(80 \mathrm{kcal} / \mathrm{dL})$, term infants on breast milk or formula $(67 \mathrm{kcal} / \mathrm{dL})$. Time points of measurement : after reaching full enteral feeding, at term and two further time points until a maximum of 6 months of corrected age. BC was measured by Dual energy X-ray absorptiometry (QDR 1500; Hologic). R software (GAMLSS) used for construction of growth curves.

Results Length of preterms remain shorter than terms, both increases linearly at $0.7 \mathrm{~cm} / \mathrm{wk}$. Median FM/FFM in preterm is $500 \mathrm{~g} / 2450 \mathrm{~g}$ (40 wks) and $1700 \mathrm{~g} / 4500 \mathrm{~g}$ (60 wks) compared to $430 \mathrm{~g} / 2790 \mathrm{~g}$ (40 wks) and $2400 \mathrm{~g} / 4700 \mathrm{~g}$ (60 wks) for term. Preterm FMI centiles are higher than terms till 45-50 wks corrected. Preterm FFMI increases progressively till 40 wks, then remains constant over time like terms (Figure 1).

Conclusion Growth pattern for preterm vary from term, justifying their higher nutritional requirement to support rapid FFM growth initially. FM being inverse of FFM, length normalised indices rather than percentages allow independent assessment of growth in each body compartment, while compensating for difference in body size of term and preterm infants.

\section{P0-0460 IDENTIFYING TRAJECTORIES FOR HEALTHY POSTNATAL GROWTH OF PRETERM INFANTS}

${ }^{1} \mathrm{P}$ Raja, ${ }^{1} \mathrm{~N}$ Rochow, ${ }^{2} \mathrm{~S}$ Goettler, ${ }^{3} \mathrm{~A}$ Jahn, ${ }^{4} \mathrm{~S}$ Seigel, ${ }^{5} \mathrm{D}$ Campbell, ${ }^{3} \mathrm{M}$ Heckmann, 2. Poeschl, ${ }^{1} \mathrm{C}$ Fusch. ${ }^{1}$ Pediatrics, McMaster University, Hamilton, Canada; ${ }^{2}$ Pediatrics, University of Heidelberg, Heidelberg, Germany; ${ }^{3}$ Pediatrics, University of Greifswald, Greifswald, Germany; ${ }^{4}$ Pediatrics, St. Joseph's Healthcare, Hamilton, Canada; ${ }^{5}$ Pediatrics, St. Michael's Hospital, Toronto, Canada
Background Growth of preterm infants should follow intrauterine rates. Postnatal loss of extracellular fluid shifts growth trajectories to a percentile below that in-utero. Which 'new' trajectory a preterm infant should adjust to after completed postnatal adaptation is unknown.

Objective 1) To develop a model for postnatal growth trajectories of preterm infants by characterising growth of such infants which required only minimal postnatal support; 2) to predict trajectories for healthy postnatal growth in any given infant.

Methods Inclusion criteria: infants with (A) 30-35 and (B) 2429 weeks GA, admitted 2008-2012 to participating hospitals. Exclusion criteria: (A)+(B) maternal diabetes/substance use, nosocomial sepsis (positive blood culture until day of life (DoL) 21 (A) nCPAP $>3$ days, not on full enteral feeds by DoL 10, (B) mechanical ventilation on $\mathrm{DoL}>3, \mathrm{FiO} 2 \geq 0.3$ within first 21 DoL, NEC >stage 2, IVH $>2$, PVL. Models to predict body weight trajectories on DoL 14 and 21 were developed.

Results 890 infants were eligible of 6915 meeting inclusion criteria. Infants had maximum weight loss by DoL 5 , regained birth weight by DoL 11 and showed stable growth parallel to intrauterine percentiles during DoL 7-21. Surprisingly the new trajectory was independent from GA with a z-score difference from birth of (A) $-0.96 \pm 0.75$ and $(\mathrm{B})-0.88 \pm 0.67$ at DoL14. Linear regression models predicted weight at DoL $14(\mathrm{R} 2=0.88)$ and $21(\mathrm{R} 2=0.82)$. Conclusions 1) The study provides robust estimates of ideal postnatal growth trajectories for preterm infants. 2) The impact on long-term outcome using these trajectories for nutritional adjustment needs to be assessed, ideally in an RCT.

\section{P0-0461 HEART RATE VARIABILITY IN FULL-TERM NEONATES WITH HYPOXIC ISCHAEMIC ENCEPHALOPATHY}

RM Goulding, NJ Stevenson, DM Murray, V Livingstone, GB Boylan. Neonatal Brain Research Group INFANT Centre, University College Cork, Cork, Ireland 